\title{
Insulin resistance is associated with poorer verbal fluency performance in women
}

\author{
Laura L. Ekblad ${ }^{1,2}$ (1) Juha O. Rinne ${ }^{1,3}$ - Pauli J. Puukka ${ }^{4}$ - Hanna K. Laine ${ }^{5,6}$. \\ Satu E. Ahtiluoto ${ }^{7}$. Raimo O. Sulkava ${ }^{7}$ - Matti H. Viitanen ${ }^{5,8}$ • Antti M. Jula ${ }^{4}$
}

Received: 31 March 2015 / Accepted: 10 July 2015 / Published online: 15 August 2015

(C) Springer-Verlag Berlin Heidelberg 2015

\begin{abstract}
Aims/hypothesis Type 2 diabetes is an independent risk factor for cognitive decline. Insulin resistance occurring during midlife may increase the risk of cognitive decline later in life. We hypothesised that insulin resistance is associated with poorer cognitive performance and that sex and $A P O E^{*} E 4$ might modulate this association.

Methods The association of insulin resistance and $A P O E^{*} E 4$ genotype on cognitive function was evaluated in a nationwide Finnish population-based study $(n=5,935$, mean age 52.5 years, range $30-97$ years). HOMA-IR was used to measure insulin resistance. Cognitive function was tested by word-list learning, word-list delayed-recall, categorical verbal fluency and simple and visual-choice reaction-time tests. Linear regression analysis was used to determine the association between HOMA-IR and the results of the cognitive tests. Results Higher HOMA-IR was associated with poorer verbal fluency in women $(p<0.0001)$ but not in men $(p=0.56)$.
\end{abstract}

Laura L. Ekblad

llekbl@utu.fi

Turku PET Centre, University of Turku and Turku University Hospital, P.O. Box 52, 20521 Turku, Finland

2 Turku Health Care Centre, Turku, Finland

3 Division of Clinical Neurosciences, Turku University Hospital, Turku, Finland

4 National Institute for Health and Welfare (THL), Turku, Finland

5 Turku City Hospital, University of Turku, Turku, Finland

6 Department of Medicine, University of Turku, Turku, Finland

7 University of Eastern Finland, Kuopio, Finland

8 Clinical Geriatrics, Karolinska Institutet, Karolinska University Hospital, Huddinge, Sweden
Higher HOMA-IR was also associated with poorer verbal fluency in $A P O E^{*} E 4$-negative individuals $(p=0.0003)$, but not in $A P O E^{*} E 4$ carriers $(p=0.28)$. Furthermore, higher HOMA-IR was associated with a slower simple reaction time in the whole study group $(p=0.02)$.

Conclusions/interpretation To our knowledge, this is the first comprehensive, population-based study, including both young and middle-aged adults, to report that female sex impacts the association of HOMA-IR with verbal fluency. Our study was cross-sectional, so causal effects of HOMA-IR on cognition could not be evaluated. However, our results suggest that HOMA-IR could be an early marker for an increased risk of cognitive decline in women.

Keywords Alzheimer's disease $\cdot A P O E^{*} E 4$ genotype · Categorical verbal fluency $\cdot$ Cognition $\cdot$ HOMA-IR $\cdot$ Insulin resistance $\cdot$ Sex difference

$\begin{array}{ll}\text { Abbreviations } \\ \text { BDI } & \text { Beck's depression inventory } \\ \text { PET } & \text { Positron emission tomography } \\ \text { RT } & \text { Simple reaction time } \\ \text { VC } & \text { Visual-choice reaction time } \\ \text { WLDR } & \text { Word-list delayed recall } \\ \text { WLL } & \text { Word-list learning } \\ \text { WMH } & \text { White matter hyperintensity }\end{array}$

\section{Introduction}

Type 2 diabetes and Alzheimer's disease are both common conditions. The number of individuals suffering from these diseases continues to rise, and type 2 diabetes is an independent risk factor for cognitive decline and Alzheimer's disease 
$[1,2]$. In addition, insulin resistance is linked to cognitive decline through different mechanisms [3, 4]. Insulin has specific effects on the brain, and insulin transportation through the blood-brain barrier is actively regulated $[4,5]$. In peripheral hyperinsulinaemia, insulin transportation to the brain is reduced [6]. Reduced levels of insulin in the brain can directly contribute to cognitive decline and the pathophysiology of Alzheimer's disease in multiple ways; for example, by downregulating the insulin-degrading enzyme in the brain [5]. This enzyme plays an important role in $\beta$-amyloid clearance, and reduced levels of insulin-degrading enzyme lead to excess $\beta$-amyloid, the hallmark of Alzheimer's disease pathology in the brain.

Central nervous system insulin resistance has become an important point of interest in recent studies. Brain insulin resistance could be a triggering factor in the development of Alzheimer's disease [7]. Brain insulin resistance has been studied in humans using magnetoencephalography. This showed an increase in brain cortical activity in lean but not in obese individuals during a two-step euglycaemichyperinsulinaemic clamp when compared with a saline infusion. The increase in brain cortical activity correlated positively with peripheral insulin sensitivity, indicating that insulin resistance is present in the central nervous system [8]. In addition, in an ex vivo study, the response of insulin receptor signalling pathways to insulin signalling was remarkably reduced in the brain tissues of participants with Alzheimer's disease, thereby indicating that brain insulin resistance is present in those with Alzheimer's disease [9].

Administration of intranasal insulin may be a promising therapeutic strategy for patients with Alzheimer's disease $[10,11]$. In cognitively healthy young volunteers, intranasal insulin has been shown to have a positive effect on multiple cognitive domains, including verbal memory [12], spatial memory [13] and working memory [14]. Previous studies indicate a sex difference in the response to intranasal insulin treatment $[14,15]$.

A few studies have attempted to examine the association between cognitive function and insulin resistance. In a crosssectional analysis of 1,898 individuals representing a familybased cohort from a genetically isolated population, insulin resistance was related to executive function in women only [16]. In a Swedish community-based, cross-sectional study of 285 cognitively healthy elderly volunteers [17], HOMA-IR [18] was inversely associated with verbal fluency performance in both men and women. In that study, higher HOMA-IR levels were also associated with a reduced volume of grey matter in the temporal lobe brain regions that support language ability. In a study using positron emission tomography (PET), HOMA-IR was associated with reduced cerebral glucose metabolism in the frontal, parietotemporal and cingulate regions in cognitively normal adults [19]. These brain areas are commonly affected in patients with prodromal Alzheimer's disease [20].
The apolipoprotein E $\varepsilon 4$ allele $\left(A P O E^{*} E 4\right)$ is a wellknown risk factor for Alzheimer's disease [21] and for progression of impaired memory in patients with mild cognitive impairment [22]. $A P O E^{*} E 4$ may play a role in modulating the risk of Alzheimer's disease in patients with diabetes [23]. In addition, the positive effect of intranasal insulin on cognition seems to apply only to $A P O E^{*} E 4$-negative individuals $[15,24]$. To date, the associations among $A P O E^{*} E 4$, insulin resistance and cognitive performance remain unknown.

Based on previous findings, we hypothesised that HOMAIR, a measure of insulin resistance, could be an independent risk factor for poorer cognitive performance, and that $A P O E^{*} E 4$ and sex would modulate this risk. To test this hypothesis, we evaluated the associations of HOMA-IR, $A P O E^{*} E 4$ genotype, sex and cognitive performance in a representative nationwide sample of the Finnish adult population with a wide age range (30-97 years).

\section{Methods}

Study design and participants The data for this study were acquired from the Health 2000 Survey, a comprehensive, nationwide, population-based examination survey that was conducted by the Finnish National Institute for Health and Welfare in 2000-2001. The survey randomly selected 8,028 individuals aged 30 years or older from the Finnish population register using a two-stage stratified cluster sampling procedure. The participation rate was $84 \%(n=6,770)$ for the health examination proper or the examination at home $[25,26]$.

The study was approved by the Ethics Committee for Epidemiology and Public Health in the hospital district of Helsinki and Uusimaa, Finland. Written informed consent was obtained from all participants.

Our study cohort was obtained from the population that fasted for $4 \mathrm{~h}$ or more $(n=6,366)$. People living in institutions were not included. We excluded individuals on insulin treatment or taking unknown diabetes medication; those with missing plasma insulin or glucose values; and those who did not complete the cognitive tests. The selection process is shown in Fig. 1. The final study population consisted of 5,935 individuals, with a mean age of 52.5 years (range 30 97 years); $55 \%$ were women.

Clinical examination BP was measured in a sitting position from the right arm with a standard mercury manometer (Mercuro 300; Speidel \& Keller, Jungingen, Germany), and the average of two measurements was used for analyses. Blood samples were drawn and the duration of fasting time was recorded. $A P O E$ genotype was assessed in 5,549 individuals who gave their written consent for DNA sampling. 


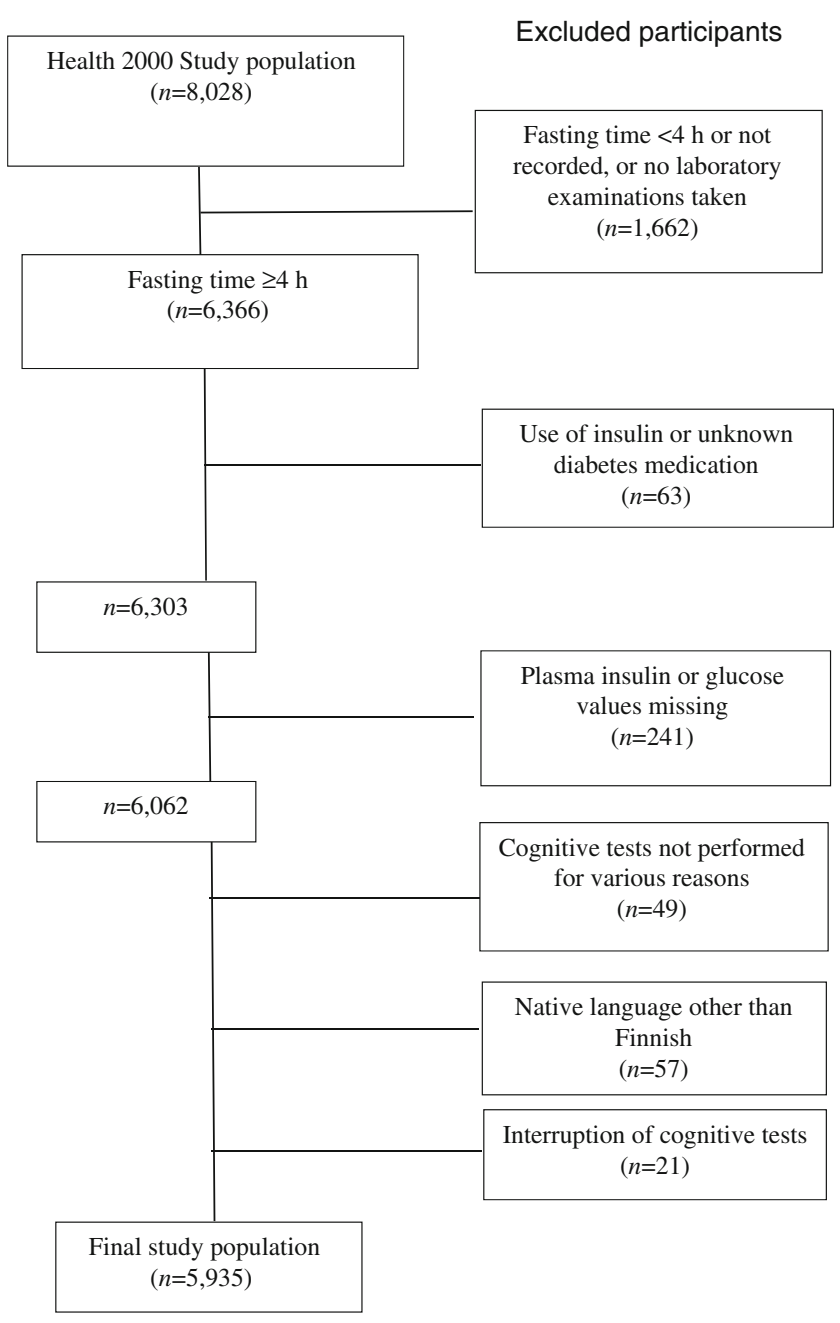

Fig. 1 The study population selection process

Cholesterol values were determined using a CHOD PAP test (Olympus System Reagent, Hamburg, Germany), HDLcholesterol using a HDL-C Plus test (Roche Diagnostics, Mannheim, Germany), triacylglycerols using a GPO PAP test (Olympus System Reagent) and glucose using a hexokinase test (Olympus System Reagent). Serum insulin was determined using a microparticle enzyme immunoassay (Abbott Laboratories, Dainabot, Tokyo, Japan). $\mathrm{HbA}_{1 \mathrm{c}}$ was determined using an immunoturbidimetric method (Hemoglobin A1c assay; Abbott Laboratories). HOMA-IR was calculated as described previously [18]. Non-HDL-cholesterol was determined using the formula: total cholesterol-HDL-cholesterol. APOE genotyping was performed using the MassARRAY System (Sequenom, San Diego, CA, USA) with a modified protocol, which has been described elsewhere [27].

Physical activity was assessed by asking participants how often, in their free time, they exercised for at least $30 \mathrm{~min}$ and vigorously enough to cause sweating and mild breathlessness. The results were classified as follows: $1=\mathrm{a}$ few times a year or less, $2=$ two or three times a month, $3=$ once a week, $4=$ two or three times a week, $5=$ four to six times a week, $6=$ daily.
Cognitive tests The participants were tested for verbal fluency, encoding and retaining verbal material according to the Finnish version of the CERAD (Consortium to Establish a Registry for Alzheimer's Disease) test battery [26, 28, 29]. In the verbal fluency test, participants were asked to list as many animals as possible during $1 \mathrm{~min}$. In the word-list learning (WLL) test, ten words were shown to the participants. Participants were asked to read the words aloud, memorise them and repeat the words they remembered within $90 \mathrm{~s}$. This was repeated twice more, if needed. We used the sum score of the words learned after three rounds as an assessment of memory encoding. The range for this test was $0-30$ points. If the participant remembered all ten words after one round, the result was counted as the full 30 points. After 5 min (during which the reaction-time tests were performed), participants were again asked to recall all ten words; the number of recalled words was the score for the word-list delayed-recall (WLDR) test.

We examined global cognition and executive function with a computerised reaction-time test (Good Response; Metitur, Jyväskylä, Finland). The test is described in detail elsewhere $[26,30,31]$. Briefly, participants were instructed to react as quickly as possible to a light that appeared on a panel by shifting the index finger of their writing hand from the waiting switch to the switch that had lit up. Participants performed two different tests: a simple reaction-time (RT) test and a visualchoice reaction-time (VC) test. In the RT test, the same switch lit up 12 times at random intervals. The performance on this test represents the function of global cognition [32]. In the VC test, the lights lit up 12 times at different parts of the panel at random intervals. This test requires more processing and is thought to represent the function of the pre-frontal cortex, and this could be considered part of the executive function of the brain [33]. We used the mean values of reaction time on the RT test as an assessment of global cognition and the mean values of reaction time on the $\mathrm{VC}$ test as an assessment of executive function.

A total of 277 participants did not complete the RT test and 438 did not complete the VC test. Those who were unable to complete these tests were older (the mean age of those who completed both tests was 51.3 years, while the mean age of those who did not complete the VC test was 68.1 years) and had higher HOMA-IR values (median 1.62 vs 1.98).

Statistical analysis To evaluate the possible effect of fasting time on HOMA-IR, we compared the HOMA-IR values of participants with a fasting time of 4-6 h (median HOMA-IR 1.55), 6-8 h (1.59), 8-10 h (1.52) and more than $10 \mathrm{~h} \mathrm{(1.80)}$ by the non-parametrical Kruskal-Wallis test and compared each pair using the Steel-Dwass method. There was no statistically significant difference between the groups, except for a significantly higher HOMA-IR for the group with the longest fasting time. Therefore, we assumed that including those who 
had fasted for between 4 and $10 \mathrm{~h}$ would not give falsely greater HOMA-IR values and interfere with the interpretation of HOMA-IR.

The characteristics of our study population are shown as means \pm SDs and ranges (Table 1). Sex differences were assessed with Student's $t$ test for continuous variables and $\chi^{2}$ test for categorical variables. The Wilcoxon test was used to analyse variables with skewed distributions (i.e. fasting time, RT and VC, Beck's depression inventory [BDI]). Before further analyses, the skewed distributions of HOMA$\mathrm{IR}$, triacylglycerols, BDI score and $\mathrm{HbA}_{1 \mathrm{c}}$ were corrected using logarithmic transformation $\left(\log _{e}\right)$. Pearson's correlation was used to examine correlations of risk factors for cognitive decline with verbal fluency, WLL and WLDR scores. Spearman's correlation was used to analyse RT and VC.

Multiple linear regression analysis was used to identify determinants that were independently associated with poorer cognitive performance. Adjustments were made for HOMAIR, age, education, sex, systolic BP, BMI, non-HDL- and HDL-cholesterol, triacylglycerols, $A P O E^{*} E 4$ genotype, BDI score and self-reported physical activity. The skewed distributions of RT and VC were corrected using logarithmic transformation $\left(\log _{e}\right)$.
The interaction terms 'sex $\times$ HOMA-IR', 'APOE* $E 4 \times$ HOMA-IR' and 'age $\times$ HOMA-IR' on the cognitive test results were analysed in a fully adjusted regression model. The interaction terms were considered significant at $p<0.1$. There was a significant ' $\mathrm{sex} \times$ HOMA-IR' interaction for the verbal fluency $(p=0.099)$ and WLL $(p=0.07)$ scores. The interaction of 'age $\times$ HOMA-IR' was significant only for the WLL score $(p=0.06)$. The interaction of ' $A P O E^{*} E 4 \times$ HOMA-IR' was significant for the verbal fluency $(p=0.09)$ and WLDR $(p=$ 0.097 ) scores. For clarity, the results are presented separately for men and women in the tables. The results for the whole study group are reported in the text for the test results for which the interaction term 'sex $\times$ HOMA-IR' was not significant (i.e. WLDR, RT, VC). Data were analysed using SAS 9.3 (SAS Institute, Cary, NC, USA).

\section{Results}

When comparing our study group and the excluded individuals $(n=2,093)$ with a Student's $t$ test, we found that the study participants were younger (mean age: 52.5 vs 59.0 years,

Table 1 Characteristics of the study population

\begin{tabular}{|c|c|c|c|c|c|}
\hline \multirow[t]{2}{*}{ Characteristic } & \multicolumn{2}{|l|}{ Men } & \multicolumn{2}{|l|}{ Women } & \multirow[t]{2}{*}{$p$ value } \\
\hline & $\mathrm{Mean} \pm \mathrm{SD}^{\mathrm{a}}$ & Range & Mean $\pm \mathrm{SD}^{\mathrm{a}}$ & Range & \\
\hline$n(\%)$ & $2,673(45.0)$ & & $3,262(55.0)$ & & \\
\hline Age, years & $51.5 \pm 14.0$ & $30-97$ & $53.4 \pm 15.3$ & $30-94$ & $<0.0001$ \\
\hline Education, years $(n=5,885)$ & $11.2 \pm 4.0$ & $0-31$ & $11.3 \pm 4.2$ & $0-33$ & 0.12 \\
\hline Systolic BP, mmHg $(n=5,924)$ & $135 \pm 19$ & $89-227$ & $133 \pm 22$ & $89-240$ & 0.002 \\
\hline BMI, $\mathrm{kg} / \mathrm{m}^{2}(n=5,929)$ & $27.1 \pm 4.1$ & $16.5-51.9$ & $26.8 \pm 5.1$ & $12.3-52.5$ & 0.004 \\
\hline $\mathrm{HbA}_{1 \mathrm{c}}, \%(\mathrm{mmol} / \mathrm{mol})(n=5,844)$ & $5.38 \pm 0.55(35 \pm 6.0)$ & $3.1-11.2(10-99)$ & $5.21 \pm 0.52(33 \pm 5.7)$ & $3.6-11.3(16-100)$ & 0.002 \\
\hline HOMA-IR $(n=5,935)$ & $2.47 \pm 6.03$ & $0.19-289.94$ & $2.02 \pm 1.76$ & $0.18-29.49$ & $<0.0001$ \\
\hline Fasting time, h:min & $9: 27$ & $4: 00-39: 25$ & $9: 25$ & $4: 00-35: 04$ & 0.35 \\
\hline HDL-cholesterol, $\mathrm{mmol} / \mathrm{l}(n=5,935)$ & $1.20 \pm 0.33$ & $0.23-3.01$ & $1.44 \pm 0.38$ & $0.49-3.02$ & $<0.0001$ \\
\hline Non-HDL-cholesterol, $\mathrm{mmol} / \mathrm{l}(n=5,935)$ & $4.77 \pm 1.15$ & $1.22-11.04$ & $4.48 \pm 1.13$ & $1.47-9.68$ & $<0.0001$ \\
\hline Triacylglycerols, mmol/1 $(n=5,935)$ & $1.79 \pm 1.25$ & $0.4-16.6$ & $1.40 \pm 0.72$ & $0.4-9.7$ & $<0.0001$ \\
\hline$A P O E^{*} E 4$ genotype ${ }^{\mathrm{c}} n(\%)(n=5,549)$ & $806(32.2)$ & & $975(32.0)$ & & 0.92 \\
\hline Verbal fluency score $(n=5,935)$ & $23.5 \pm 7.4$ & $0-52$ & $23.9 \pm 7.1$ & $3-48$ & 0.04 \\
\hline WLL score $(n=5,934)$ & $19.8 \pm 4.4$ & $0-30$ & $21.1 \pm 4.6$ & $0-30$ & $<0.0001$ \\
\hline WLDR score $(n=5,935)$ & $6.7 \pm 2.0$ & $0-10$ & $7.2 \pm 2.1$ & $0-10$ & $<0.0001$ \\
\hline $\mathrm{RT}, \mathrm{s}(n=5,658)$ & $0.32 \pm 0.08$ & $0.19-1.12$ & $0.34 \pm 0.09$ & $0.2-1.23$ & $<0.0001$ \\
\hline $\mathrm{VC}, \mathrm{s}(n=5,497)$ & $0.47 \pm 0.13$ & $0.25-1.63$ & $0.49 \pm 0.14$ & $0.25-1.58$ & $<0.0001$ \\
\hline BDI score $(n=5,793)$ & $6.13 \pm 6.54$ & $0-46$ & $7.81 \pm 7.14$ & $0-55$ & $<0.0001$ \\
\hline Physical activity score $(n=5,791)$ & $3.54 \pm 1.48$ & $1-6$ & $3.64 \pm 1.47$ & $1-6$ & 0.009 \\
\hline
\end{tabular}

${ }^{\text {a }}$ Unless otherwise stated

${ }^{\mathrm{b}}$ Differences between the sexes were assessed using Student's $t$ test for continuous variables and the $\chi^{2}$ test for categorical variables. The Wilcoxon test was used for fasting time and the RT and VC tests because of a skewed distribution

${ }^{\mathrm{c}} A P O E^{*} E 4$ was considered positive for participants with one or two $\varepsilon 4$ alleles 
$p<0.0001)$ and more educated (11.2 vs 9.3 years of education, $p<0.0001$ ).

The characteristics for men and women are shown in Table 1. There were significant sex differences for all variables except fasting time $(p=0.35)$, education $(p=0.12)$ and $A P O E^{*} E 4$ status $(p=0.92)$.

Univariate correlates of cognitive functioning Higher age and shorter time in education were associated with poorer verbal fluency, WLL and WLDR, and with increased RT and VC (Table 2). There was a strong inverse correlation between age and years of education $(r=-0.51, p<0.0001)$. Higher HOMA-IR, $\mathrm{HbA}_{1 \mathrm{c}}$, systolic BP, BMI, triacylglycerol and BDI scores and lower HDL-cholesterol levels (more consistently among women) were associated with poorer cognitive functioning in both sexes. Higher non-HDL-cholesterol and lower physical activity levels were associated with poorer cognitive functioning in women only (Table 2).

Multivariate correlates of cognitive functioning The final model of our linear regression analysis is shown in Table 3. The explanatory value of HOMA-IR alone (adjusted $R^{2}$ ) was, for men and women, respectively: $0.7 \%$ and $3.5 \%$ on the verbal fluency test; $0.6 \%$ and $4.0 \%$ on the WLL test; $0.6 \%$ and $2.7 \%$ on the WLDR test; $1.2 \%$ and $2.2 \%$ on the RT test; and $0.9 \%$ and $2.0 \%$ on the VC test. Adding age and education significantly improved the explanatory value of the model to, for men and women, respectively: $15.8 \%$ and $23.2 \%$ on the verbal fluency test; $35.5 \%$ and $36.8 \%$ on the WLL test; $32.4 \%$ and $33.2 \%$ on the WLDR test; $10.3 \%$ and $16.9 \%$ on the RT test; and $20.7 \%$ and $29.7 \%$ on the VC test (data not shown). Adding the other risk factors of cognitive decline did not improve the explanatory value of our model. However, we wanted to add these previously reported risk factors of cognitive decline to our model in order to compare the value of HOMA-IR as an explanatory variable of cognitive decline to the other risk factors. The adjusted $R^{2}$ values for our final model are shown in Table 3.

Higher HOMA-IR was highly significantly associated with poorer verbal fluency in women $(p<0.0001)$ but not in men $(p=0.56)$. Higher HOMA-IR was also associated with a slower reaction time on the RT test in the whole study group $(p=0.02)$ (data not shown). HOMA-IR was not associated with the other cognitive tests (i.e. WLL, WLDR, or VC). $A P O E^{*} E 4$ genotype was associated with lower scores on the WLL $(p=0.004)$ and WLDR $(p=0.005)$ tests in women, and with a faster response time on the RT test in men $(p=0.003)$.

$A P O E^{*} E 4$ modulated the association of HOMA-IR with the verbal fluency and WLDR scores, as the interaction term 'APOE*E4 $\times$ HOMA-IR' was statistically significant for these tests. The association of HOMA-IR with these test scores was analysed separately in $A P O E^{*} E 4$-positive and -negative individuals. HOMA-IR was associated with poorer verbal fluency in $A P O E^{*} E 4$-negative individuals $(p=0.0003)$, but not in $A P O E^{*} E 4$ carriers $(p=0.28)$. HOMA-IR was not associated with WLDR scores in either group (both $p>0.26$ ).

To compare the association of long-term glucose homeostasis and insulin resistance on cognitive function, we

Table 2 Correlations between potential risk factors for cognitive decline and various dimensions of cognitive function

\begin{tabular}{|c|c|c|c|c|c|c|c|c|c|c|}
\hline \multirow[t]{2}{*}{ Characteristic } & \multicolumn{2}{|l|}{$\mathrm{VF}$} & \multicolumn{2}{|l|}{ WLL } & \multicolumn{2}{|l|}{ WLDR } & \multicolumn{2}{|l|}{ RT } & \multicolumn{2}{|l|}{$\mathrm{VC}$} \\
\hline & Men & Women & Men & Women & Men & Women & Men & Women & Men & Women \\
\hline Age & $-0.28 * * *$ & $-0.39 * * *$ & $-0.53 * * *$ & $-0.57 * * *$ & $-0.53 * * *$ & $-0.55 * * *$ & $0.27 * * *$ & $0.35^{* * *}$ & $0.41 * * *$ & $0.51 * * *$ \\
\hline Education & $0.39 * * *$ & $0.46^{* * *}$ & $0.50 * * *$ & $0.51 * * *$ & $0.45 * * *$ & $0.47 * * *$ & $-0.21 * * *$ & $-0.28 * * *$ & $-0.31 * * *$ & $-0.37 * * *$ \\
\hline HOMA-IR & $-0.08 * * *$ & $-0.19 * * *$ & $-0.08 * * *$ & $-0.20 * * *$ & $-0.08 * * *$ & $-0.17 * * *$ & $0.10 * * *$ & $0.13 * * *$ & $0.10^{* * *}$ & $0.14 * * *$ \\
\hline Systolic BP & $-0.15 * * *$ & $-0.24 * * *$ & $-0.23 * * *$ & $-0.33^{* * *}$ & $-0.23 * * *$ & $-0.33 * * *$ & $0.13 * * *$ & $0.22 * * *$ & $0.17 * * *$ & $0.31 * * *$ \\
\hline BMI & $-0.04 *$ & $-0.12 * * *$ & $-0.04 *$ & $-0.16^{* * *}$ & $-0.04 *$ & $-0.14 * * *$ & $0.06^{* *}$ & $0.14 * * *$ & $0.05^{* *}$ & $0.15^{* * *}$ \\
\hline HDL-cholesterol & $0.05 * *$ & $0.09 * * *$ & 0.02 & $0.11 * * *$ & 0.02 & $0.09 * * *$ & $-0.08 * * *$ & $-0.10 * * *$ & $-0.07 * * *$ & $-0.07 * * *$ \\
\hline Non-HDL-cholesterol & -0.01 & $-0.14 * * *$ & 0.02 & $-0.20 * * *$ & 0.01 & $-0.19 * * *$ & 0.04 & $0.11 * * *$ & 0.01 & $0.18 * * *$ \\
\hline Triacylglycerols & -0.02 & $-0.17 * * *$ & -0.02 & $-0.24 * * *$ & -0.01 & $-0.22 * * *$ & $0.07 * * *$ & $0.15^{* * *}$ & $0.05^{*}$ & $0.18^{* * *}$ \\
\hline BDI score & $-0.12 * * *$ & $-0.11 * * *$ & $-0.18 * * *$ & $-0.17 * * *$ & $-0.18 * * *$ & $-0.15 * * *$ & $0.09 * * *$ & $0.16^{* * *}$ & $0.15 * * *$ & $0.15^{* * *}$ \\
\hline Physical activity & 0.01 & $0.05^{* *}$ & -0.02 & 0.03 & -0.03 & 0.01 & -0.02 & -0.01 & $0.05 *$ & 0.01 \\
\hline $\mathrm{HbA}_{1 \mathrm{c}}$ & $-0.12 * * *$ & $-0.22 * * *$ & $-0.18^{* * *}$ & $-0.28^{* * *}$ & $-0.17 * * *$ & $-0.27 * * *$ & $0.13^{* * *}$ & $0.20 * * *$ & $0.18^{* * *}$ & $0.25 * * *$ \\
\hline$A P O E^{*} E 4^{\mathrm{a}}$ & 1.00 & 1.00 & 1.00 & 0.99 & 1.00 & 0.98 & 0.57 & 0.76 & 0.97 & 0.67 \\
\hline
\end{tabular}

Pearson's correlation was used to analyse VF, WLL and WLDR results, while Spearman's correlation was used to analyse RT and VC results

${ }^{a} \mathrm{ORs}$ for $A P O E^{*} E 4$ per unit change for the cognitive tests used ' $A P O E^{*} E 4$ positive' vs 'APOE* $E 4$ negative'

$n=5,935$

${ }^{*} p<0.05, * * p<0.01, * * * p<0.001$

VF, verbal fluency 
Table 3 Multivariate linear regression analysis of the risk factors for cognitive decline and the cognitive tests used

\begin{tabular}{|c|c|c|c|c|c|c|c|c|c|c|}
\hline \multirow[t]{2}{*}{ Standardised estimate } & \multicolumn{2}{|l|}{$\mathrm{VF}$} & \multicolumn{2}{|l|}{ WLL } & \multicolumn{2}{|l|}{ WLDR } & \multicolumn{2}{|l|}{ RT } & \multicolumn{2}{|l|}{$\mathrm{VC}$} \\
\hline & Men & Women & Men & Women & Men & Women & Men & Women & Men & Women \\
\hline HOMA-IR & -0.02 & $-0.10 * * *$ & 0.02 & -0.02 & -0.001 & -0.004 & 0.06 & 0.04 & 0.03 & 0.01 \\
\hline Age & $-0.01 * * *$ & $-0.16^{* * *}$ & $-0.35^{* * *}$ & $-0.40 * * *$ & $-0.40 * * *$ & $-0.39 * * *$ & $0.24 * * *$ & $0.34 * * *$ & $0.39 * * *$ & $0.51 * * *$ \\
\hline Education & $0.32 * * *$ & $0.33 * * *$ & $0.32 * * *$ & $0.26^{* * *}$ & $0.23 * * *$ & $0.21 * * *$ & $-0.08 * *$ & $-0.11 * * *$ & $-0.11 * * *$ & $-0.08^{* *}$ \\
\hline Systolic BP & -0.03 & -0.008 & -0.005 & -0.007 & -0.009 & $-0.04 *$ & -0.002 & -0.01 & -0.02 & -0.02 \\
\hline BMI & 0.01 & $0.05^{*}$ & -0.02 & 0.02 & -0.01 & 0.02 & -0.02 & -0.003 & $-0.05^{* * *}$ & 0.02 \\
\hline Non-HDL-cholesterol & 0.02 & $0.05 *$ & $0.05^{*}$ & $0.07 * * *$ & 0.03 & $0.07 * * *$ & 0.001 & $-0.09 * * *$ & -0.03 & $-0.07 * *$ \\
\hline HDL-cholesterol & $0.07 * *$ & 0.03 & -0.04 & 0.03 & -0.02 & 0.03 & -0.02 & $-0.06 * *$ & -0.04 & -0.02 \\
\hline Triacylglycerols & 0.03 & 0.004 & -0.03 & -0.04 & 0.007 & $-0.05^{*}$ & 0.03 & 0.007 & 0.03 & 0.02 \\
\hline$A P O E^{*} E 4^{\mathrm{a}}$ & 0.01 & -0.001 & 0.0005 & $-0.05 * *$ & -0.006 & $-0.05 * *$ & $-0.06 * *$ & -0.0003 & -0.009 & -0.01 \\
\hline BDI score & -0.03 & -0.01 & $-0.04 *$ & $-0.03 * *$ & -0.04 & -0.02 & 0.03 & $0.07 * * *$ & $0.05^{*}$ & $0.05 *$ \\
\hline Physical activity & 0.03 & 0.03 & 0.01 & 0.51 & 0.07 & -0.004 & $-0.05^{*}$ & $-0.04 *$ & 0.009 & $-0.04 *$ \\
\hline Adjusted $R^{2 \mathrm{~b}}$ & 0.157 & 0.209 & 0.347 & 0.360 & 0.309 & 0.314 & 0.097 & 0.175 & 0.208 & 0.292 \\
\hline
\end{tabular}

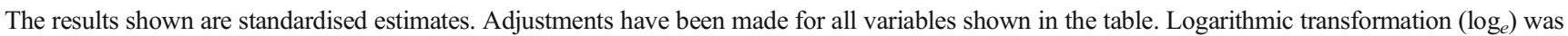
used for HOMA-IR, triacylglycerol, BDI points, and RT and VC tests in the analysis

${ }^{\mathrm{a}} A P O E^{*} E 4$ refers to a participant with one or two $\varepsilon 4$ alleles

${ }^{\mathrm{b}}$ Coefficient of determination=adjusted $R^{2}$ for the total explanatory value of our model for each cognitive test and for men and women separately $n=5,935$

$* p<0.05, * * p<0.01, * * * p<0.001$

$\mathrm{VF}$, verbal fluency

analysed $\mathrm{HbA}_{1 \mathrm{c}}$ using a similar linear regression analysis model as that for HOMA-IR. Higher $\mathrm{HbA}_{1 \mathrm{c}}$ levels were associated with a slower response time on the $\mathrm{VC}$ test in men ( $p=$ $0.04)$. Unexpectedly, $\mathrm{HbA}_{1 \mathrm{c}}$ levels were positively associated with WLL scores in women $(p=0.028)$ and with a faster response time on the VC test in women $(p=0.002)$. There was no significant association between $\mathrm{HbA}_{1 \mathrm{c}}$ levels and the results of other cognitive tests.

\section{Discussion}

Here, we show that there are significant sex and $A P O E^{*} E 4$ differences in the association of HOMA-IR with cognitive performance. Higher HOMA-IR was strongly associated with poorer performance on categorical verbal fluency tests in women only. There were no sex differences for the WLDR, RT or VC tests. Higher HOMA-IR was associated with a slower reaction time in the whole study group on the RT test. In addition, higher HOMA-IR was associated with poorer verbal fluency in participants who did not carry the $A P O E^{*} E 4$ allele, whereas this association was not seen in $A P O E^{*} E 4$-positive participants. Our final model of linear regression analysis did not explain the results of the verbal fluency test as well for men as it did for women (adjusted $R^{2}=$ $15.7 \%$ vs $20.9 \%$ ), suggesting the presence of other underlying factors that might explain the level of verbal fluency present in men. The association of HOMA-IR with verbal fluency in women was independent of the $\mathrm{HbA}_{1 \mathrm{c}}$ level of our participants. This indicates that insulin resistance might have early effects on cognition, and that these effects might be detected even before the development of hyperglycaemia and type 2 diabetes.

Several previous studies have shown that insulin resistance is associated with declines in executive function and, especially, verbal fluency $[16,17,34]$. In addition, lower levels of physical activity have been associated with poorer performance on verbal fluency tests [35], and animal studies have shown that low levels of physical activity lead to peripheral insulin resistance [36]. However, when compared with our study population, these earlier studies were significantly smaller and focused only on the elderly $[17,34,35]$ or represented a genetically isolated cohort [16]. To our knowledge, our study is the first to report a sex difference in the association between HOMA-IR and verbal fluency in a nationwide, comprehensive population that includes young adults. We did not find an association between insulin resistance and results on the VC test, which was used to measure executive function.

Previous studies have also shown that $A P O E^{*} E 4$ genotype influences the effect of intranasal insulin on cognition [15, 24]. Our results are consistent with previous findings showing that non-carriers of $A P O E^{*} E 4$ may be more sensitive to the effects of insulin on cognitive performance [15, 24]. 
Previous studies on intranasal insulin therapy have shown sex differences in the positive effect of insulin on cognition $[14,15]$. Cognitively healthy young women, but not men, have been reported to benefit from acute insulin treatment on tasks measuring verbal working memory and spatial learning [14]. In a study with participants with mild cognitive impairment and Alzheimer's disease, both men and women performed better on delayed story recall after taking insulin $20 \mathrm{IU} /$ day for 4 months, but only men benefited from a $40 \mathrm{IU}$ dose [15]. Categorical verbal fluency was not tested in these studies. Although a difference in verbal ability has been reported in favour of women [37], this does not seem to apply to categorical verbal fluency $[38,39]$. In the current study, we found a statistically significant difference between men and women $(p=0.04)$ in verbal fluency scores. This does not, however, explain the greater effect of HOMA-IR on women. A decline in categorical verbal fluency has previously been shown in a longitudinal study in women with a high risk for coronary heart disease according to the Framingham Cardiac Risk Score [40]. Insulin resistance was not assessed in that study.

Our observational study is limited, as we cannot yet establish causality. There are, however, several potential pathways that can explain our findings. Categorical verbal fluency represents the function of the lateral and inferior temporal lobe regions, whereas first-letter-based verbal fluency relies on the function of the prefrontal cortex [41]. Consistent with the localisation of verbal fluency in the temporal regions, a previous study of elderly participants found that HOMA-IR was negatively associated with categorical verbal fluency and temporal lobe grey matter volume [17]. In the Baltimore Longitudinal Study of Aging, midlife glucose intolerance was related to a greater longitudinal decrease in regional cerebral blood flow (measured by ${ }^{15} \mathrm{O}$-water PET scanning) in the frontal, parietal and temporal cortices [42]. In addition, in a fluorodeoxyglucose PET study, HOMA-IR was associated with reduced cerebral glucose metabolism in frontal, parietotemporal and cingulate regions in cognitively normal adults [19].

One possible explanation for the observed sex difference in our study could be the previously demonstrated sex difference in brain white matter hyperintensities (WMHs). Vascular changes related to insulin resistance, as demonstrated in PET and MRI studies [17, 19, 42], might lead to white matter lesions in the brain [43]. Individuals with the metabolic syndrome have more WMHs than those who are metabolically healthy [44]. Women have more WMHs than men, and this difference is greater for periventricular WMHs than for deep WMHs [45]. These results could explain the sex difference and the association between insulin resistance or vascular risk factors and poorer verbal fluency performance in both this and a previous epidemiological study [40].
In a study by Makino and colleagues, small periventricular hyperintensities on cerebral MRI correlated with poorer performance in a categorical verbal fluency test for participants with amnestic mild cognitive impairment, but not for those with Alzheimer's disease [46]. Amnestic mild cognitive impairment can be considered a prodromal phase of Alzheimer's disease [47]. The study's authors suggested that this difference in cognitive performance could be caused by the greater significance of periventricular WMHs in the early stages of Alzheimer's disease and prodromal Alzheimer's disease (i.e. amnestic mild cognitive impairment). We suggest that insulin resistance could be a significant risk factor for WMHs in women, and that WMHs could have a role in modulating the cognitive decline related to insulin resistance. Unfortunately, we were unable to perform brain MRI scans on our study volunteers, and so could not directly assess the effects of insulin resistance on brain white matter lesions.

White matter lesions seem to play a role in the pathology of Alzheimer's disease [48, 49]. Therefore, the association between insulin resistance and cognitive function could indicate that insulin resistance is a risk factor for Alzheimer's disease. This risk could be modulated by several mechanisms, such as vascular damage, white matter lesions, direct mechanisms of insulin resistance on the accumulation of $\beta$-amyloid and functional changes in regional cerebral blood flow and glucose metabolism, or by a combination of these processes.

Our study had limitations. First, the cross-sectional study design did not allow us to evaluate the causal effects of HOMA-IR on cognition. Second, the fasting times of participants varied and this might have increased the HOMA-IR values. Before deciding to use more than $4 \mathrm{~h}$ as a cut-off point for the required fasting time, we analysed the distribution of HOMA-IR according to the fasting time of participants. The HOMA-IR values were not higher for participants who had been fasting $4-10 \mathrm{~h}$ compared with those who had been fasting for more than $10 \mathrm{~h}$. Thus, we are confident that the fasting time did not influence our results. There was no difference between the average fasting times for men and women.

There were significant differences between our study group and the excluded individuals with respect to age and education. The differences between these groups are likely to represent an overall willingness and ability to participate in clinical surveys, because the group of excluded individuals included those who did not attend the actual health examination. The reaction-time tests were apparently difficult to complete, especially for elderly individuals. This might explain the lack of an association between insulin resistance and the VC test, which was used to measure executive function.

The strength of our study is in the representative, nationwide, population-based design. Compared with previous studies, the inclusion of young adults was another strength. We were also able to use a number of risk factors for cognitive decline as covariates in our analysis. 
In conclusion, we have shown that insulin resistance is associated with poorer verbal fluency in women, which strengthens previous findings showing that insulin has sexspecific effects on cognition. Although verbal fluency is not the most sensitive measure with which to identify early cognitive decline in adults, it has a good association with insulin resistance in women and with the brain regions that are negatively influenced by insulin resistance [17]. The inclusion of young and middle-aged adults in our study means that our findings suggest that the association of insulin resistance with poorer cognition is present years before the onset of more severe cognitive defects. In the future, longitudinal studies should be conducted to explore the possibility of a causal relationship between insulin resistance and cognition.

Acknowledgements We thank K. Silander, M. Kaunisto and the staff of the Technology Centre, FIMM, University of Helsinki, for the APOE genotyping work. The protocol is available upon request. We thank all the volunteers who participated in the Health 2000 Study for their generous contribution. R. M. Badeau (University of Turku Language Centre, Tur$\mathrm{ku}$, Finland) is acknowledged for English-language content editing.

Funding This study was funded by Finnish Governmental Research Funding, the Betania Foundation, the Sigrid Jusélius Foundation, the Uulo Arhio Foundation and the Yrjö Jahnsson Foundation.

Duality of interest The authors declare that there is no duality of interest associated with this manuscript.

Contribution statement LLE researched the data and wrote the manuscript. LLE, JOR, PJP, HKL, SEA, ROS, MHV and AMJ planned the study. JOR, PJP, HKL, SEA, ROS, MHV and AMJ critically revised and edited the manuscript for important intellectual content. PJP helped with the statistical analysis of the data. All authors approved the final version of the manuscript to be published. LLE is the guarantor of this work.

\section{References}

1. Ott A, Stolk RP, van Harskamp F, Pols HA, Hofman A, Breteler MM (1999) Diabetes mellitus and the risk of dementia: the Rotterdam study. Neurology 53:1937-1942

2. Biessels GJ, Staekenborg S, Brunner E, Brayne C, Scheltens P (2006) Risk of dementia in diabetes mellitus: a systematic review. Lancet Neurol 5:64-74

3. Hallschmid M, Schultes B (2009) Central nervous insulin resistance: a promising target in the treatment of metabolic and cognitive disorders? Diabetologia 52:2264-2269

4. Craft S, Cholerton B, Baker LD (2013) Insulin and Alzheimer's disease: untangling the web. J Alzheimers Dis 33(Suppl 1): S263-S275

5. Cholerton B, Baker LD, Craft S (2013) Insulin, cognition, and dementia. Eur J Pharmacol 719:170-179

6. Schwartz MW, Figlewicz DF, Kahn SE, Baskin DG, Greenwood MR, Porte D Jr (1990) Insulin binding to brain capillaries is reduced in genetically obese, hyperinsulinemic zucker rats. Peptides $11: 467-472$

7. Correia SC, Santos RX, Perry G, Zhu X, Moreira PI, Smith MA (2011) Insulin-resistant brain state: the culprit in sporadic Alzheimer's disease? Ageing Res Rev 10:264-273
8. Tschritter O, Preissl H, Hennige AM et al (2006) The cerebrocortical response to hyperinsulinemia is reduced in overweight humans: a magnetoencephalographic study. Proc Natl Acad Sci U S A 103:12103-12108

9. Talbot K, Wang HY, Kazi H et al (2012) Demonstrated brain insulin resistance in Alzheimer's disease patients is associated with IGF-1 resistance, IRS-1 dysregulation, and cognitive decline. J Clin Invest 122:1316-1338

10. Reger MA, Watson GS, Green PS et al (2008) Intranasal insulin improves cognition and modulates $\beta$-amyloid in early $\mathrm{AD}$. Neurology 70:440-448

11. Craft S, Baker LD, Montine TJ et al (2012) Intranasal insulin therapy for Alzheimer disease and amnestic mild cognitive impairment: a pilot clinical trial. Arch Neurol 69:29-38

12. Benedict C, Hallschmid M, Hatke A et al (2004) Intranasal insulin improves memory in humans. Psychoneuroendocrinology 29:1326-1334

13. Brünner YF, Kofoet A, Benedict C, Freiherr J (2015) Central insulin administration improves odor-cued reactivation of spatial memory in young men. J Clin Endocrinol Metab 100:212-219

14. Benedict C, Kern W, Schultes B, Born J, Hallschmid M (2008) Differential sensitivity of men and women to anorexigenic and memory-improving effects of intranasal insulin. J Clin Endocrinol Metab 93:1339-1344

15. Claxton A, Baker LD, Wilkinson CW et al (2013) Sex and ApoE genotype differences in treatment response to two doses of intranasal insulin in adults with mild cognitive impairment or Alzheimer's disease. J Alzheimers Dis 35:789-797

16. Schuur M, Henneman P, van Swieten JC et al (2010) Insulinresistance and metabolic syndrome are related to executive function in women in a large family-based study. Eur J Epidemiol 25:561-568

17. Benedict $\mathrm{C}$, Brooks SJ, Kullberg $\mathrm{J}$ et al (2012) Impaired insulin sensitivity as indexed by the HOMA score is associated with deficits in verbal fluency and temporal lobe gray matter volume in the elderly. Diabetes Care 35:488-494

18. Matthews DR, Hosker JP, Rudenski AS, Naylor BA, Treacher DF, Turner RC (1985) Homeostasis model assessment: insulin resistance and beta-cell function from fasting plasma glucose and insulin concentrations in man. Diabetologia 28:412-419

19. Baker LD, Cross DJ, Minoshima S, Belongia D, Watson GS, Craft S (2011) Insulin resistance and Alzheimer-like reductions in regional cerebral glucose metabolism for cognitively normal adults with prediabetes or early type 2 diabetes. Arch Neurol 68:51-57

20. Langbaum JB, Chen K, Lee W et al (2009) Alzheimer's Disease Neuroimaging Initiative. Categorical and correlational analyses of baseline fluorodeoxyglucose positron emission tomography images from the Alzheimer's Disease Neuroimaging Initiative (ADNI). Neuroimage 45:1107-1116

21. Corder EH, Saunders AM, Strittmatter WJ et al (1993) Gene dose of apolipoprotein E type 4 allele and the risk of Alzheimer's disease in late onset families. Science 261:921-923

22. Petersen RC, Smith GE, Ivnik RJ et al (1995) Apolipoprotein E status as a predictor of the development of Alzheimer's disease in memory-impaired individuals. JAMA 273:1274-1278

23. Peila R, Rodriguez BL, Launer LJ, Honolulu-Asia Aging Study (2002) Type 2 diabetes, APOE gene, and the risk for dementia and related pathologies: the Honolulu-Asia Aging Study. Diabetes 51:1256-1262

24. Reger MA, Watson GS, Frey WH 2nd et al (2006) Effects of intranasal insulin on cognition in memory-impaired older adults: modulation by APOE genotype. Neurobiol Aging 27:451-458

25. Aromaa A, Koskinen S (2004) Health and functional capacity in Finland. Baseline results of the Health 2000 Health Examination Survey. Publications of the National Public Health Institute, 
Helsinki, B12. Available from www.terveys2000.fi/julkaisut/ baseline.pdf, accessed 16 Jul 2015

26. Heistaro S (ed) (2008) Methodology report. Health 2000 Survey. Publications of the National Public Health Institute, Helsinki, B26. Available from www.terveys2000.fi/doc/methodologyrep.pdf, accessed 16 Jul 2015

27. Jänis MT, Siggins S, Tahvanainen E et al (2004) Active and lowactive forms of serum phospholipid transfer protein in a normal Finnish population sample. J Lipid Res 45:2303-2309

28. Morris JC, Heyman A, Mohs RC et al (1989) The Consortium to Establish a Registry for Alzheimer's Disease (CERAD). Part I. Clinical and neuropsychological assessment of Alzheimer's disease. Neurology 39:1159-1165

29. Hänninen T, Pulliainen V, Salo J et al (1999) Cognitive tests in diagnosing memory disorders and early dementia: CERAD. Suom Lääkäril 15:1967-1975 [article in Finnish]

30. Era P, Jokela J, Heikkinen E (1986) Reaction and movement times in men of different ages: a population study. Percept Mot Skills 63:111-130

31. Kronholm E, Sallinen M, Era P, Suutama T, Sulkava R, Partonen T (2011) Psychomotor slowness is associated with self-reported sleep duration among the general population. J Sleep Res 20:288-297

32. Rabbitt P (1996) Do individual differences in speed reflect "global" or "local" differences in mental abilities? Intelligence 22:69-88

33. van der Meer E, Beyer R, Horn J et al (2010) Resource allocation and fluid intelligence: insights from pupillometry. Psychophysiology 47:158-169

34. Geroldi C, Frisoni GB, Paolisso G et al (2005) Insulin resistance in cognitive impairment: the InCHIANTI Study. Arch Neurol 62:1067-1072

35. Benedict C, Brooks SJ, Kullberg J et al (2013) Association between physical activity and brain health in older adults. Neurobiol Aging 34:83-90

36. Sarvas JL, Otis JS, Khaper N, Lees SJ (2015) Voluntary physical activity prevents insulin resistance in a tissue specific manner. Physiol Rep 3, e12277

37. Miller DI, Halpern DF (2014) The new science of cognitive sex differences. Trends Cogn Sci 18:37-45

38. Brucki SM, Rocha MS (2004) Category fluency test: effects of age, gender and education on total scores, clustering and switching in
Brazilian Portuguese-speaking subjects. Braz J Med Biol Res 37:1771-1777

39. Munro CA, Winicki JM, Schretlen DJ et al (2012) Sex differences in cognition in healthy elderly individuals. Neuropsychol Dev Cogn B Aging Neuropsychol Cogn 19:759-768

40. Laughlin GA, McEvoy LK, von Muhlen D et al (2011) Sex differences in the association of Framingham cardiac risk score with cognitive decline in community-dwelling elders without clinical heart disease. Psychosom Med 73:683-689

41. Gourovitch ML, Kirkby BS, Goldberg TE (2000) A comparison of rCBF patterns during letter and semantic fluency. Neuropsychology $14: 353-360$

42. Thambisetty M, Beason-Held LL, An Y et al (2013) Impaired glucose tolerance in midlife and longitudinal changes in brain function during aging. Neurobiol Aging 34:2271-2276

43. Yates KF, Sweat V, Yau PL, Turchiano MM, Convit A (2012) Impact of metabolic syndrome on cognition and brain: a selected review of the literature. Arterioscler Thromb Vasc Biol 32:2060-2067

44. Segura B, Jurado MA, Freixenet N, Falcon C, Junque C, Arboix A (2009) Microstructural white matter changes in metabolic syndrome: a diffusion tensor imaging study. Neurology 73:438-444

45. Sachdev PS, Parslow R, Wen W, Anstey KJ, Easteal S (2009) Sex differences in the causes and consequences of white matter hyperintensities. Neurobiol Aging 30:946-956

46. Makino T, Umegaki H, Suzuki Y et al (2014) Relationship between small cerebral white matter lesions and cognitive function in patients with Alzheimer's disease and amnestic mild cognitive impairment. Geriatr Gerontol Int 14:819-826

47. Gauthier S, Reisberg B, Zaudig M et al (2006) International Psychogeriatric Association Expert Conference on mild cognitive impairment. Mild cognitive impairment. Lancet 367:1262-1270

48. Hertze J, Palmqvist S, Minthon L, Hansson O (2013) Tau pathology and parietal white matter lesions have independent but synergistic effects on early development of Alzheimer's disease. Dement Geriatr Cogn Dis Extra 3:113-122

49. Provenzano FA, Muraskin J, Tosto G et al (2013) Alzheimer's Disease Neuroimaging Initiative. White matter hyperintensities and cerebral amyloidosis: necessary and sufficient for clinical expression of Alzheimer disease? JAMA Neurol 70:455-461 